\title{
Vertical Handover Management for VoIP Session over Broadband Wireless Networks
}

\author{
Muhammad Niswar ${ }^{1}$, Shigeru Kashihara ${ }^{2}$, Suguru Yamaguchi ${ }^{2}$ \\ ${ }^{1}$ Department of Electrical Engineering, Faculty of Engineering, Hasanuddin University, Makassar, Indonesia \\ ${ }^{2}$ Graduate School of Information Science, Nara Institute of Science and Technology, Nara, Japan \\ Email: niswar@unhas.ac.id
}

Received April 22, 2013; revised May 24, 2013; accepted June 14, 2013

Copyright (C) 2013 Muhammad Niswar et al. This is an open access article distributed under the Creative Commons Attribution License, which permits unrestricted use, distribution, and reproduction in any medium, provided the original work is properly cited.

\begin{abstract}
Today, wireless LAN (IEEE802.11g/n) has been the dominant wireless network that can provide high data rates in a limited coverage area. While emerging mobile WiMAX (IEEE802.16e) can provide a wireless broadband access to mobile users in a wide coverage area. These two different technolgies will co-exist while complementing each other in some regions, hence, a mobile node (MN) with dual interfaces traverses and executes many handovers (HOs) between 802.11g and 802.16e. Meanwhile, there is a huge demand for Voice over IP (VoIP) service over wireless networks. However, VoIP is a delay and loss sensitive application, hence, VoIP session is more likely to be deteriorated during HO between $802.11 \mathrm{~g}$ and $802.16 \mathrm{e}$. In order to maintain quality of VoIP session during HO, we proposed Vertical Handover Management (VHM) for VoIP session that focuses on HO initiation and decision strategy according to the wireless link condition and congestion state of wireless networks. The VHM exploits request to send (RTS) retries and round trip time (RTT) between an MN and an access point in an 802.11g interface as well as a carrier to interference noise ration (CINR) level and MN's queue length of an 802.16e interface as HO triggers. We then conducted simulation experiments to evaluate the effectiveness of our proposed VHM using QualNet 4.5. Our simulation results show that our proposed VHM can preserve the quality of VoIP session during such HOs.
\end{abstract}

Keywords: Handover Management; Handover Triggers; Single/Bi-Casting; 802.11; 802.16e; VoIP

\section{Introduction}

Wireless networks will consist of various wireless network technologies and mobile users will traverse several coverage areas of different wireless access technologies. Recently, mobile WiMAX (IEEE 802.16e) has gained serious attention as a means of providing wireless broadband access to mobile users in a wide area, and it provides QoS for various applications. On the other hand, IEEE $802.11 \mathrm{~g}$ has already been widely used to provide high data rates in a limited area. Therefore, in the near future, since $802.11 \mathrm{~g}$ and $802.16 \mathrm{e}$ networks are intermingled while complementing each other, a mobile node (MN) will be likely to execute many handovers (HOs) between $802.11 \mathrm{~g}$ and $802.16 \mathrm{e}$ networks with different IP subnets (e.g. two networks that administrated by two different ISPs).

Meanwhile, there is a huge demand for Voice over IP (VoIP) service over wireless networks. However, delivering VoIP over wireless has many challenges because VoIP is a delay and packet loss sensitive application. In a mobile and wireless environment, typically, two main factors degrade VoIP quality over the wireless network: 1) degradation of wireless link quality; and 2) wireless network congestion. First, because an MN moves freely, the communication quality degrades due to the fluctuation of the wireless link condition. Second, as VoIP is a bi-directional communication, an access point (AP) of $802.11 \mathrm{~g}$ and a base station (BS) of 802.16e become a bottleneck with the increase of VoIP calls. When an MN traverses several APs and BSs, an MN needs to detect the degradation of VoIP quality and $\mathrm{HO}$ to the best available wireless network.

In order to detect the degradation of wireless link quality as well as a congestion of wireless network in a heterogeneous wireless environment, we cannot only rely on one $\mathrm{HO}$ trigger such as Carrier-to-Interference plus Noise Ratio (CINR) level. This is because in vertical HO, we cannot directly compare the CINR level of different wireless technologies since each technology has different characteristics in terms of MAC and physical layer. For instance, they might have the same CINR level but differ 
in data rate. Therefore, it is a challenge to develop a vertical HO management (VHM) considering suitable HO triggers in order to achieve seamless HO between two different types of wireless networks. In this paper, to preserve VoIP quality during $\mathrm{HO}$, we then propose VHM for VoIP over the intermingled 802.11g and 802.16e networks considering the wireless link condition and congestion state of networks.

\section{Related Work}

So far, several studies on the vertical HO schemes have been conducted. References in $[1,2]$ proposed an algorithm for vertical $\mathrm{HO}$ between $3 \mathrm{G}$ and WLAN. Authors in [1] use the Received Signal Strength (RSS) with estimated lifetime to execute the HO. On the other hand, authors in [2] use a dynamic RSS threshold that calculated and compared with the current RSS to execute the HO. Reference [3] proposed a link trigger generation method based on RSS. The author proposed a smart triggering scheme by determining link status transition including Link-Up (LU), Link-Coming-Up (LCU), LinkGoing-Down (LGD) and Link-Down (LD). Each link state have RSS threshold for transition. These link status will trigger the SIP session mobility protocol to execute the HO. Reference [4] proposed a cross-layer based polynomial regression predictive RSS approach with marcov decision process for optimal selection for HO in heterogeneous network. The proposed scheme consists of two primary phases, i.e., the phase of predictive RSS with a hysteresis and the phase of determining optimal target network in which to $\mathrm{HO}$ using marcov decision process. The author evaluated the proposed method using numerical analysis and compared various performance metrics including number of vertical $\mathrm{HO}$ and network throughput. Author described the proposed algorithm through mathematical analysis and evaluated through simulation. The proposed method can avoid the ping-pong effect using predictive RSS with hysteresis. Reference [1-4] schemes exploit RSS as an HO trigger, which cannot represent the received signal quality because it is only indicate the strength of received signal excludes noise, interference, and other channel effects.

Reference [5] proposed an algorithm for vertical HO between 802.11 and 802.16 hybrid networks exploiting data rate and channel occupancy as HO triggers and implemented them on top of the MAC layer. The author uses downlink Signal to Interference Noise Ratio (SINR) for wireless connectivity trigger as well as data rate and network load for performance trigger. The author validated the proposed scheme in two scenarios, i.e., single$\mathrm{MN}$ moves from WiFi-WiMAX-WiFi and system capacity evaluation. The simulation scenario limited to longlived FTP application for movement scenario. There is no evaluation for real-time application such as VoIP.

Authors in [6] introduced a mobile relay node as an intermediate node between 802.11 devices and 802.16. base station (BS) to convert 802.16 signal received from BS to 802.11 devices. This approach is inefficient because it requires additional intermediate node among 802.11 access point (AP), 802.16e BS and MN.

The most recognized study is Media Independent Handover (MIH) that was proposed by the IEEE 802.21 working group [7]. $\mathrm{MIH}$ is intended to facilitate $\mathrm{HO}$ and interoperability of various other network technologies. However, deployment of MIH requires additional layers to the existing protocol stack of mobile device and infrastructure networks leading to cost inefficiency. Therefore, in this paper, we focus on an end-to-end VHM that only requires some modification on end-node, and then our proposed method aims to preserve VoIP quality during $\mathrm{HO}$ between networks that managed by different organization.

\section{Handover Initiation}

$\mathrm{HO}$ initiation is a process to triggering $\mathrm{HO}$ according to some parameters/specific conditions. Since we intend to develop a multi-homed MN-initiated HO scheme where $\mathrm{MN}$ has two different wireless interfaces (IFs), i.e., $802.11 \mathrm{~g}$ and $802.16 \mathrm{e}$ networks, we need to determine the HO triggers for each different IF that can be obtained at the MN side. Our proposed VHM aims to preserve VoIP quality during $\mathrm{HO}$; hence, we need to consider several $\mathrm{HO}$ triggers indicating both wireless link condition and congestion state in both 802.11g and 802.16e networks. The following subsections describe our proposed $\mathrm{HO}$ triggers and the motivation behind selecting them.

\subsection{Handover Triggers for 802.11}

As VoIP is a bi-directional communication, AP becomes a bottleneck with the increase of VoIP calls. That is, VoIP packets to MNs are liable to experience large queuing delay or packet loss due to increase in queue length or buffer overflow in the AP buffer because each MN and AP has almost the same priority level of frame transmission by following the CSMA/CA scheme. In our previous study [8], we found that Request-to-Send (RTS) frame retries and Round-Trip Time (RTT) between MN and AP can be potential $\mathrm{MN}$-initiated $\mathrm{HO}$ triggers for $802.11 \mathrm{~g}$. We briefly describe these triggers in the following subsections.

\subsubsection{Frame Retries}

In the IEEE802.11 standard, a sender confirms a successful transmission by receiving an ACK frame in response to the transmitted data frame. When a data or ACK frame is lost, the sender periodically retransmits the 
same data frame until achieving a successful transmission or reaching a predetermined retry limit. When frame retries reach the retry limit, the sender treats the data frame as a lost packet.

Our previous study [9] shows that frame retries can promptly and reliable detect the wireless link degradation with compared to RSS. However, current WLANs employ a multi-rate function that can change the transmission rate according to wireless link condition. If the transmission rate is dropped by the multi-rate function, a more robust modulation type is selected and thus data frame retries are further decreased. As a result, an $\mathrm{MN}$ cannot properly detect the degradation of wireless link quality only from data frame retries in multi-rate WLANs. We then consider RTS frame retries as an alternative trigger for data frame retries. As an RTS frame is always transmitted at the lowest rate (e.g., $6 \mathrm{Mb} / \mathrm{s}$ in $802.11 \mathrm{a} / \mathrm{g}$ and $1 \mathrm{Mb} / \mathrm{s}$ in $802.11 \mathrm{~b}$ ), an MN can appropriately detect the change of wireless link quality. In our previous study [8], we found that an RTS retry ratio of less than 0.6 satisfies adequate VoIP call quality, and we suggest it as a threshold value for initiating HO in this study.

\subsubsection{AP Queue Length}

With the increase of VoIP calls in a WLAN, the AP queue length increases because all traffic to and from the WLAN has to go through the AP. The 802.11 CSMA/CA mechanism provides the AP and MNs with the same number of transmission opportunities. Suppose we have number of MNs (Nmn) transmiting upstream traffic, the MNs have an $N m n /(N m n+A P)$ share of bandwidth (BW) while the AP has only a $1 /(N m n+A P)$ share of $\mathrm{BW}$ for transmitting downstream traffic. Moreover, the AP typically needs to send more packets than the $\mathrm{MN}$; hence, each packet routed to the $\mathrm{MN}$ (downlink transmission) and queued in the AP buffer may experience a large queuing delay or packet loss due to asymmetry of BW share. Consequently, the queuing delay and the packet loss due to buffer overflow severely affect the VoIP quality of MNs. However, the IEEE802.11 (a/b/g/n) standard unfortunately does not provide a mechanism that can inform MNs of the AP queue length. Therefore, to preserve VoIP quality, an MN needs to detect the congestion of the AP by itself. In our previous study [8], we found the significance of the AP queue length and proposed a method to estimate AP queue length based on RTT between MN and AP (W-RTT). We found that W-RTT < $200 \mathrm{~ms}$ satisfies adequate VoIP call quality and we suggest it as a threshold value for initiating HO.

\subsection{HO Triggers for $\mathbf{8 0 2 . 1 6}$}

802.16e supports high data rates and multi service types; hence, it is a strong contender for wireless broadband access technology to support real-time applications such as VoIP over wireless networks. Although 802.16e supports multiple service types, often only Best Effort (BE) service type is made available at the initial phase of deployment. The BE scheme has no classification and priority to delay and/or loss sensitive applications; hence, the VoIP application must contend with various types of applications for obtaining transmission opportunity in 802.16e. Since we deal with a VoIP application which is bi-directional communication, we need to consider some metrics indicating both downlink and uplink transmission condition. We need to determine the HO triggers that can be obtained at the $\mathrm{MN}$ side.

In this study, we propose a combined use of two metrics, i.e., downlink CINR for indicating downlink transmission as well as the wireless link quality and MN's queue length for indicating uplink transmission and as well as the congestion state of the 802.16 e network. We describe the motivation behind selecting these metrics as an $\mathrm{HO}$ trigger for $\mathrm{MN}$-initiated $\mathrm{HO}$ of the 802.16e. We describe these metrics in the following subsections.

\subsubsection{CINR Level}

Many HO technologies employ RSS as an HO trigger, which can indicate the wireless link condition. RSS provides a simple indication of how strong the signal is at the receiver front end. However, the received signal excludes noise, interference, and other channel effects (e.g., multi-path fading and shadowing). Therefore, a high RSS does not always mean that the channel and signal quality are good. Instead, it gives an indication of whether a strong signal is present in the channel of interest. On the other hand, the CINR provides information on how strong the desired signal is compared to the interferer (or noise, or interference-plus-noise). In this study, an MN exploits a series of instantaneous CINR values that are used to derive the mean of the CINR using the moving mean method.

$$
\mu \operatorname{CINR}[k]=\frac{1}{N_{m}} \sum_{i=0}^{N_{m}} \mu \operatorname{CINR}[k-i]
$$

where, $\mu C I N R[k]$ is the mean of CINR during the $k$-th measurement and $N_{m}$ is the maximum number of measurement. We conduct a simulation experiment to investigate the performance of VoIP over 802.16e and the behavior of downlink CINR and MN's queue length using QualNet 4.5 [10]. We employ Mean Opinion Score (MOS) to assess the VoIP quality. MOS is defined in E-model standardized by ITU-T [11]. MOS provides a subjective numerical indication of the perceived quality of conversational voice call that ranges from 1 (lowest quality) to 5 (highest quality). MOS is determined based on R-factor which mainly sum of delay and loss impairment [12]. Normally, MOS $>3.60$ means an adequate 
VoIP call quality.

We set an urban environment in our simulation scenario by introducing a Rayleigh fading model to meet our scenario (see Figure 1(A)). In this simulation experiment, we employ VoIP codec of G.711, which are commonly used in the VoIP system where G.711 sends a packet of 160 bytes every $20 \mathrm{~ms}$. The detail of simulation parameters describes in Table 1. Through simulation experiments, we aim to determine a certain level of threshold for downlink CINR and MN's queue length to meet the requirement of an adequate VoIP call quality.

Figure 2 shows the relationship between Downlink MOS and CINR when an MN moves away from BS. Since the relationship starts to scatter when CINR below $50 \mathrm{~dB}$ due to the fluctuation of wireless link condition, we employ a local regression smoothing method (loess method) to grasp their trend and estimate the best fit of CINR level and MOS over the distance shown as a red curve. The red curve shows that CINR level above $26 \mathrm{~dB}$ satisfies an adequate VoIP call. Therefore, we can set the

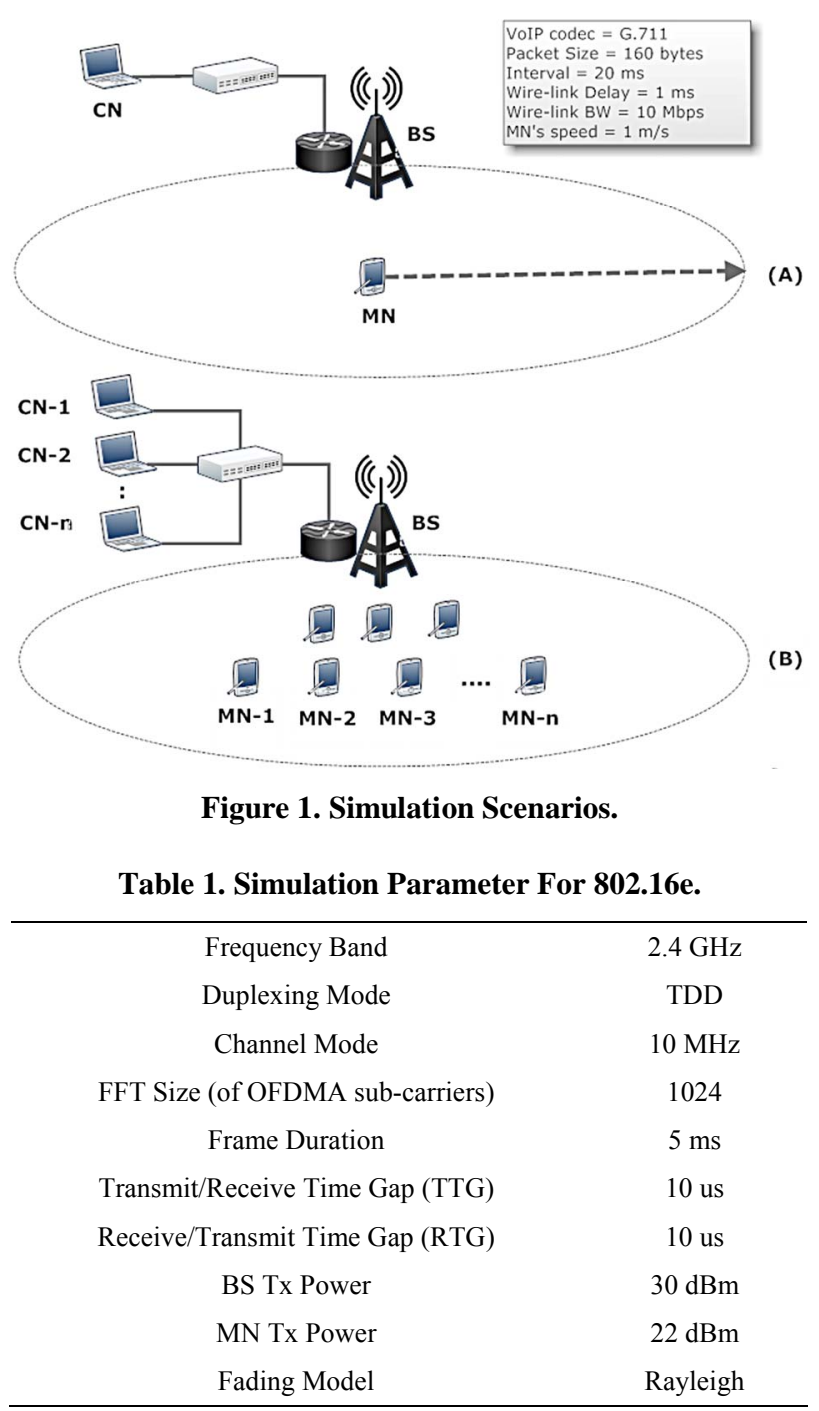

CINR level of $26 \mathrm{~dB}$ as a threshold for initiating $\mathrm{HO}$ in this study.

\subsubsection{MN's Queue Length}

In the $802.16 \mathrm{e}$ standard, BW allocation is thoroughly managed by a BS, in which the downlink BW is managed by a BW scheduler at the BS. On the other hand, uplink BW is allocated by the BS through the BW request and uplink grant procedures. In particular to uplink transmission, as shown in Figure 3, the BS allocates BW to the MNs for the purpose of making BW requests by providing a $\mathrm{BW}$ request contention slot in UL-MAP. Then, the MN transmits the BW requests in response to the allocated time slot. After the MN obtains an Uplink

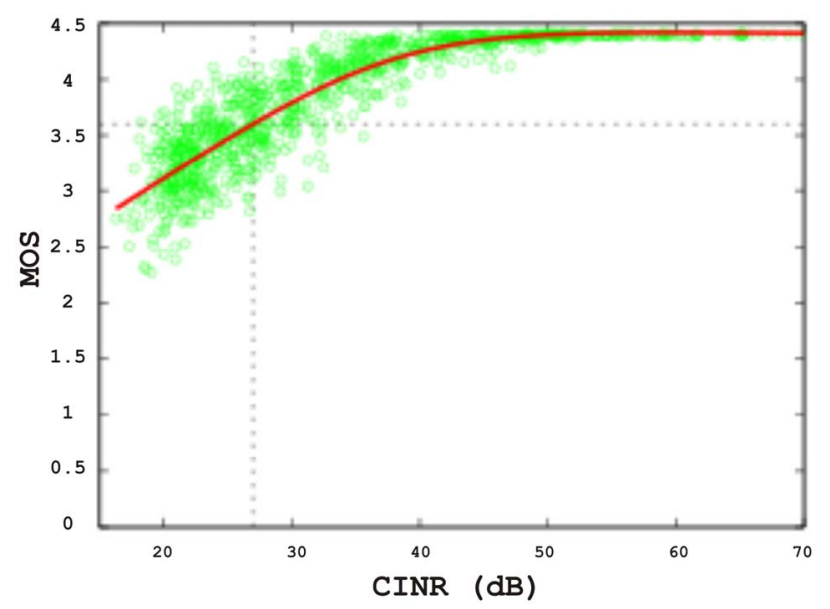

Figure 2. MOS vs. CINR.

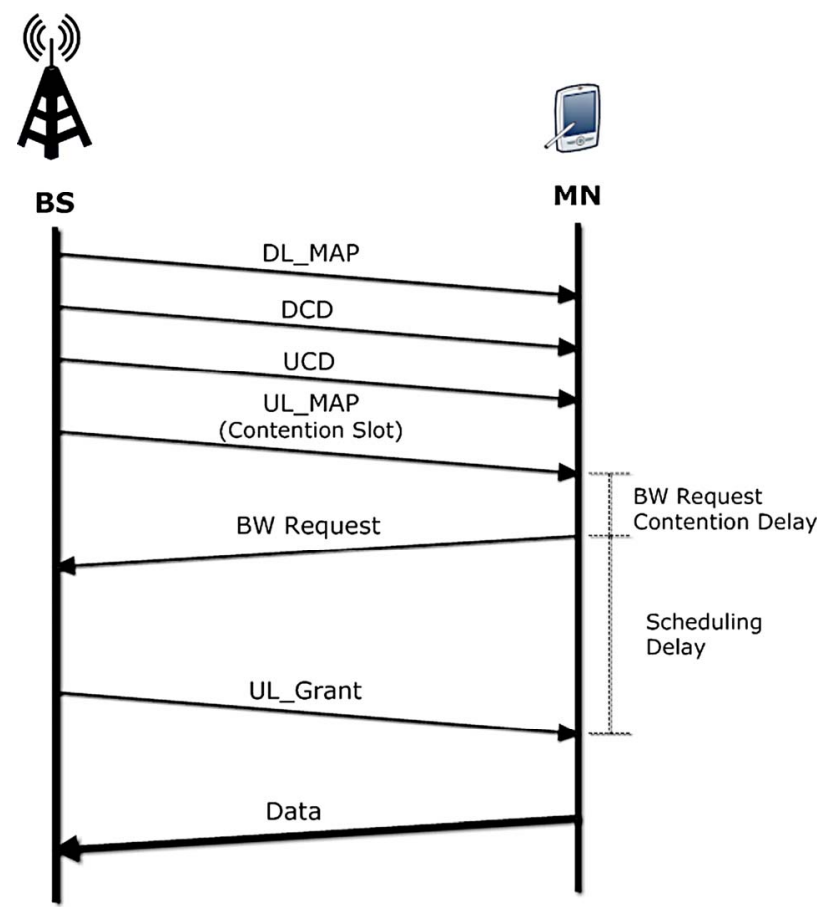

Figure 3. Uplink Transmission of 802.16e. 
Grant, it can then transmit its data with the allocated uplink grants. So, we can sum up the total end-to-end uplink delay $\left(T_{U L-E T E}\right)$ as follows:

$$
T_{\text {UL-ETE }}=T_{\text {BWreq }}+T_{\text {Sch }}+T_{\text {queue }}+T_{o}
$$

where $T_{B \text { req }}$ and $T_{S c h}$ are BW request contention and BS scheduling delay, respectively. $T_{\text {queue }}$ represents the queuing delay in the transmission buffer. $T_{o}$ represents additional delays, e.g., transmission delays over wireless link and VoIP codec delay. This BW request contention and scheduling delay may becomes longer with the increase in the number of MNs in a serving BS, which contends the BW request slot in the UL-MAP.

The increase of $\mathrm{BW}$ request contention and scheduler delay cause the increase of data on the queue length in the $\mathrm{MN}$ for transmission (MN's queue length), thereby leading to a degradation of uplink communication quality. We conduct a simulation experiment to investigate the relationship between the number of MNs establishing VoIP calls and MN's queue length. In this simulation scenario, we randomly locate from one to $30 \mathrm{MNs}$ within a coverage area of the single BS. Each MN communicates with a Correspondent Node (CN) using VoIP. Figure 1(B) shows the simulation model where every $\mathrm{MN}$ moves randomly at a speed of $1 \mathrm{~m} / \mathrm{s}$ within a coverage area of the single BS.

Figure 4 shows the average uplink and downlink VoIP quality as well as the average of MN's queue length over the number of MNs in the single BS. The figure shows that uplink VoIP quality decreases as the number of MNs in the single BS increases. On the other hand, downlink VoIP quality is kept at an adequate VoIP quality even if the number of VoIP calls increases. Moreover, the MN's queue length increases as the number of MNs establishing VoIP calls in the single BS increases. From the figure, we can see that the bottleneck of the 802.16e network affects only flows from the $\mathrm{MN}$ to $\mathrm{BS}$, i.e., uplink transmission. This bottleneck causes the uplink latency, which is mainly raised from the uplink BW request contention and scheduling delay. This uplink latency causes an increase of the MN's queue length, thereby leading to the degradation of uplink communication quality.

In terms of accommodation of MNs establishing VoIP call in the single BS, Figure 5 shows that a single BS can simultaneously accommodate $20 \mathrm{MNs}$ VoIP calls. In this simulation experiment, we aim to determine the threshold level of MN's queue length to meet the requirement of an adequate VoIP call quality condition in the $802.16 \mathrm{e}$ network. Therefore, we show the relationship among MN's queue length, uplink VoIP quality and uplink latency in Figure 6. From this figure, we can see that the G.711 VoIP application has a tolerable uplink end-to-end delay up to $260 \mathrm{~ms}$.

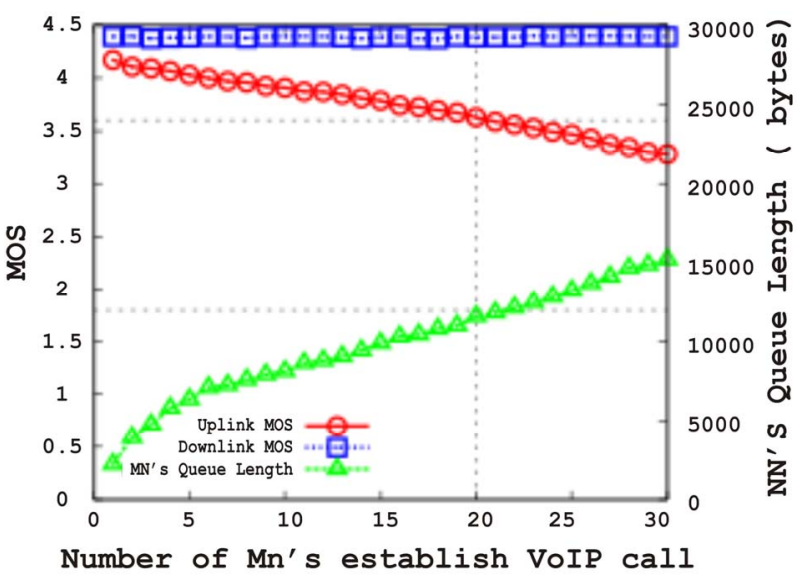

Figure 4. Relationship among MOS, MN's QL.

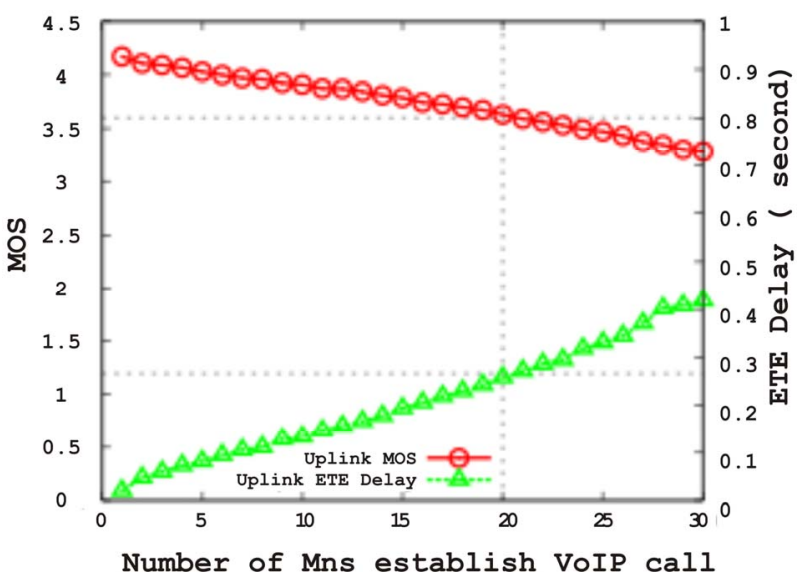

Figure 5. Relationship among MOS, ETE Delay and MN's QL.

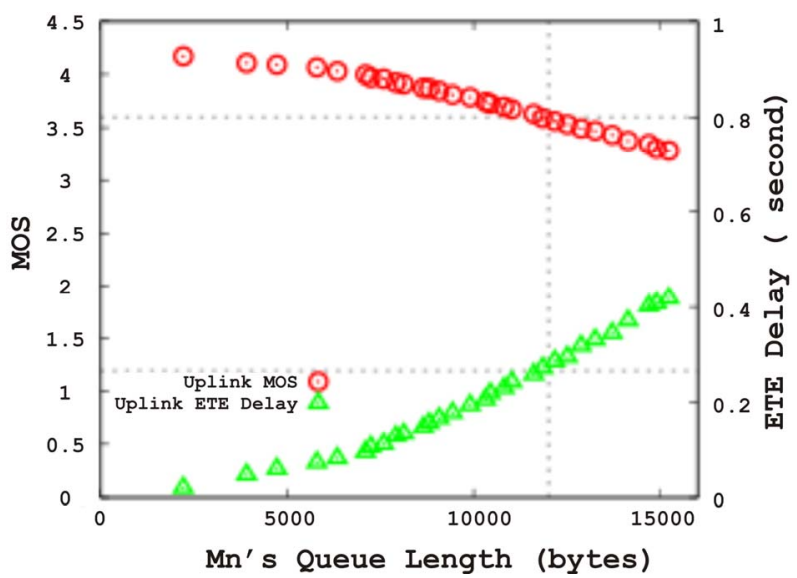

Figure 6. Relationship among MOS, ETE Delay and \# of VoIP calls.

Since we are interested in MN's queue length, we can see from Figure 6 that MN's queue lengths of less than 12,000 bytes meet the requirement for adequate VoIP call quality. Therefore, we set the MN's queue length threshold of 12,000 bytes that can be a reference for ini- 
tiating the $\mathrm{HO}$.

\section{Proposed VHM Architecture}

We propose a VHM for intermingled $802.11 \mathrm{~g}$ and $802.16 \mathrm{e}$ networks. Our proposed VHM is implemented on the transport layer of the MN and obtains effective HO triggers from a low layer using a cross-layer approach as shown in Figure 7.

\subsection{VHM Features}

In our study, we consider an MN has two IFs, i.e., $802.11 \mathrm{~g}$ and $802.16 \mathrm{e} \mathrm{IFs}$, and each IF is assigned single IP address with different IP subnet. The MN has dual IFs connected to two point of attachment, i.e., BS and AP. The MN establishes VoIP call with the CN. One or both endpoints $(\mathrm{CN}$ or $\mathrm{MN})$ can be multi-homed and multiaddressed but we here focus on that only MN is multihomed in this study. Utilizing multi-homing function can enhance the session preservation between $\mathrm{MN}$ and $\mathrm{CN}$. The main purpose of utilizing the multi-homing function is that an MN may use its two IFs either alternatively or simultaneously in order to achieve seamless HO. An MN has a choice to select the best IF in order to preserve an on-going VoIP session while changing the point of attachment. The VHM selects the transmission mode, i.e., single/bi-casting, based on RTS retries and W-RTT of the 802.11g IF as well as CINR level and MN's queue length of the $802.16 \mathrm{e}$ IF as HO triggers. Our VHM is mainly focused on proper $\mathrm{HO}$ initiation according to wireless link and congestion of network condition.

\subsection{Single/Bi-Casting Transmission Mode}

As our proposed VHM employs multihoming similar to $[8,9]$ in order to enhance the VoIP session presservation

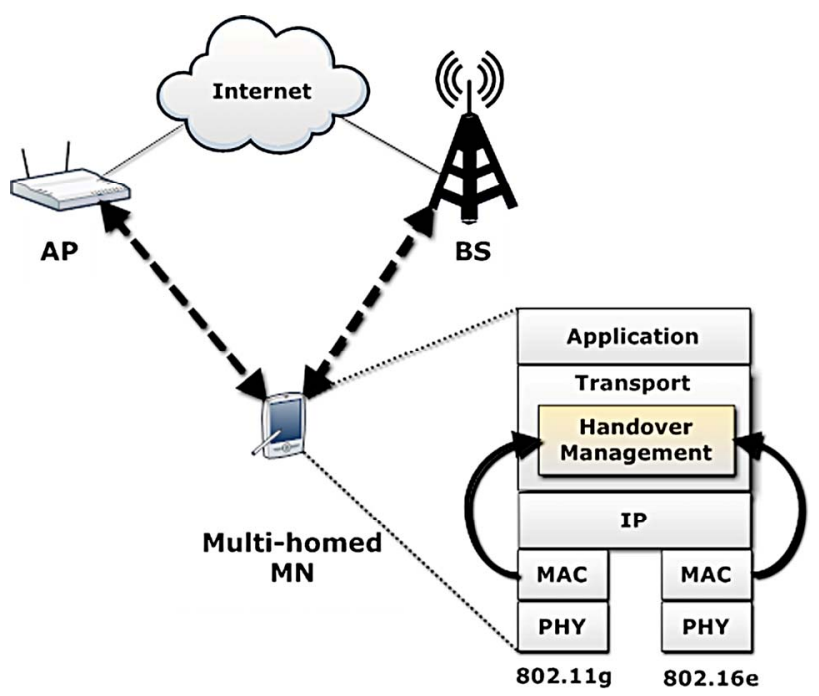

Figure 7. VHM Architecture. between $\mathrm{MN}$ and $\mathrm{CN}$, the VHM must properly switches between single-casting and bi-casting modes in response to wireless network condition. Single-casting mode means that an MN communicates with a $\mathrm{CN}$ using only one IF. Bi-casting, on the other hand, means that an $\mathrm{MN}$ sends duplicated packets to a $\mathrm{CN}$ through two IFs for supporting soft-HO. Bi-casting is useful for avoiding packet loss and investigating both $802.11 \mathrm{~g}$ and $802.16 \mathrm{e}$ IFs condition when an MN changes its IF during communication; hence, it achieves more reliable VoIP communication during HO.

Pseudo-code 1 shows an algorithm of switching to bicasting when an MN is located in an overlap area of 802.11g and 802.16e (AP and BS). Basically, our algorithm first examines the wireless link condition by observing RTS retry (rts_retry) of 802.11g IF and mean CINR level (cinr) of $802.16 \mathrm{e}$ IF. After that it examines the congestion state of wireless network by observing W-RTT (w-rtt) of 802.11g IF and MN's queue length (mn_queue) of 802.16e IF. For example, when the MN's initial transmission mode is single-casting via $802.11 \mathrm{~g} \mathrm{IF}$, the VHM monitors the rts_retry to detect the wireless link quality. If the rts_retry exceeds threshold rts_thr (0.6) [6], the MN switches transmission mode to bi-casting; otherwise, it monitors $w$-rtt to detect the congestion of $802.11 \mathrm{~g}$ network. If $w$-rtt exceeds threshold rtt thr $(200 \mathrm{~ms})$ [6], the MN switches transmission mode to bi-casting; otherwise, it stays in single-casting via $802.11 \mathrm{~g}$ IF. When the MN's initial transmission mode is single-casting via $802.16 \mathrm{e} \mathrm{IF}$, the MN monitors downlink $\operatorname{cinr}$ to detect the wireless link quality. If $\operatorname{cinr}$ is less than cinr thr $(26 \mathrm{~dB})$, the MN switches transmission mode to bi-casting; otherwise, it monitors the mn_queue to detect the congestion of $802.16 \mathrm{e}$ network. If mn queue exceeds the threshold queue_thr (12,000 bytes), the MN switches transmission mode to bi-casting; otherwise, it stays in single-casting via $802.16 \mathrm{e}$ IF.

When bi-casting is applied, the MN monitors all $\mathrm{HO}$ triggers from both IFs and compares them. As shown in Pseudo-code 2, an algorithm of switching to single-casting works as follows. An MN first monitors $\mathrm{HO}$ triggers for indicating wireless link condition and follow by congestion state of wireless network. First of all, an MN monitors and evaluates link state $\mathrm{HO}$ triggers, i.e., rts rety and cinr, of $802.11 \mathrm{~g}$ and $802.16 \mathrm{e}$ IFs, respectively. If both triggers still exceed and fall below the threshold for $r t s \_t h r(0.6)$ and cinr_thr $(26 \mathrm{~dB})$, respectively, the MN stays in bi-casting mode. If rts_retry exceeds the threshold while the cinr is still above threshold, the $\mathrm{MN}$ then switches to the single-casting via 802.16e IF; otherwise, it switches to single-casting via $802.11 \mathrm{~g} \mathrm{IF}$.

When both triggers still exceed and fall below threshold for $r t s_{-} t h r$ and cinr_thr, respectively, the MN then monitors the congestion state $\mathrm{HO}$ triggers of both $802.11 \mathrm{~g}$ 
BEGIN

IF Single-casting if: $802.11 \mathrm{~g}$

READ Link State HO Triggers FROM if:802.11g

IF rts retry $\leq$ rts thr THEN

READ Congestion State HO Triggers FROM if:802.11g

IF w-rtt $\leq$ rtt thr THEN

Single-casting if: $802.11 \mathrm{~g}$

ELSE Bi-casting

EN-

DIF

ELSE

Bi-casting

ENDIF

ELSE IF Single-casting if:802.16e

READ Link State HO Triggers FROM if:802.16e

IF cinr $\geq$ cinr thr THEN

READ Congestion State HO Triggers FROM if:802.16e

IF mn queue $\leq$ queue thr THEN

ELSE

Single-casting if:802.16e

Bi-casting

ENDIF ELSE

Bi-casting

ENDIF ENDIF

END

Pseudo-code 1. Switching from single-to-bi-casting.

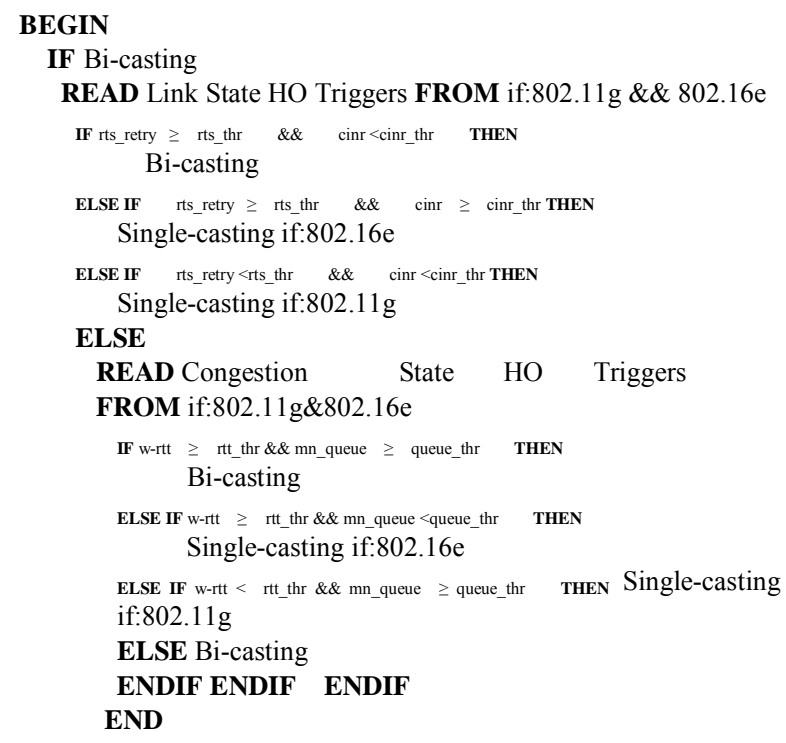

Pseudo-code 2. Switching from bi-to-bi/single-casting.

and 802.16e IFs, i.e., w-rtt and mn_queue, respectively. If both congestion state $\mathrm{HO}$ triggers exceed the threshold, the MN stays in bi-casting mode. If $w$-rtt of $802.11 \mathrm{~g} \mathrm{IF}$ exceeds the threshold while mn queue of 802.16e IF are below threshold, then, $\mathrm{MN}$ switches to the single-casting via $802.16 \mathrm{e} \mathrm{IF}$; otherwise, it switches to single-casting via $802.11 \mathrm{~g} \mathrm{IF}$. When both triggers fall below threshold, the MN switches back to the initial transmission mode that is the same mode as when the MN established a connection in the first place. When both networks remain congested, the MN continues in bi-casting leading to more congestion in both networks. In this situation, we need to extend our proposed VHM with congestion control mechanism to deal with congestion of both networks. Since we focus on HO initiation and decision in this study, we leave this topic for future investigation.

\section{Simulation Experiments and Results}

We conduct simulation experiments to investigate the effectiveness of our proposed VHM using QualNet 4.5 [10]. We evaluate our proposed VHM when degradation of wireless link occurs due to the movement of $\mathrm{MN}$ as well as when congestion of wireless network occurs due to increase of number of MNs sending VoIP traffic. In this simulation experiment, we employ VoIP codec of G.711, which is commonly used in VoIP systems where G.711 sends a packet of 160 bytes every $20 \mathrm{~ms}$. The details of simulation parameters describes in Tables 1 and 2 for $802.16 \mathrm{e}$ and $802.11 \mathrm{~g}$, respectively.

Our proposed VHM can be applied in a condition where an $802.16 \mathrm{e}$ network cell overlays an $802.11 \mathrm{~g}$ network cell; hence, an MN located in the 802.11g network cell can associate with the $802.16 \mathrm{e}$ network. We assume that both IFs are in standby mode. Therefore, the MN does not need to perform network acquisition including network scanning and ranging for $802.11 \mathrm{~g}$ and $802.16 \mathrm{e}$, respectively. Therefore, we neglect the delay caused by network acquisition.

\subsection{Performance Evaluation of HO Based on Link State HO Triggers}

This simulation experiment aims to investigate whether our proposed VHM can initiate $\mathrm{HO}$ and decide the transmission mode according to link state $\mathrm{HO}$ triggers, i.e., RTS retry of $802.11 \mathrm{~g}$ IF and CINR level of $802.16 \mathrm{e}$ IF. Figures 8(A) shows the simulation model to evaluate the proposed HO algorithms. An MN, which is establishing a VoIP call with a CN, moves from the $802.11 \mathrm{~g}$ area to the $802.16 \mathrm{e}$ area at $1 \mathrm{~m} / \mathrm{s}$, and vice versa.

In terms of VoIP quality, we can see that our proposed method can obtain average MOS values of 4.06 (Figure 9(a)) and 4.286 (Figure 9(b)) for uplink and downlink, respectively, when an MN moves from $802.11 \mathrm{~g}$ to $802.16 \mathrm{e}$. From Figure 9(a), the average uplink MOS is a bit decreased compared to downlink MOS due to uplink latency of $802.16 \mathrm{e}$, which is mainly raised from the uplink BW request contention and scheduling delays.

Table 2. Simulation Parameter for 802.11g.

\begin{tabular}{cc}
\hline Supported Data Rate (Mbps) & $6,9,12,18,24,36,48,54$ \\
Tx Power (dBm) & $5.0,5.1,6.7,7.9,8.1,9.3,10.6,10.1$ \\
SIFS & 16 us \\
Slot Time & 9 us \\
CWmin, CWmax & 15,1023 \\
\hline
\end{tabular}


On the other hand, when an MN moves from 802.16e to $802.11 \mathrm{~g}$, average MOS values are 4.29 (Figure 10(a)) and 4.28 (Figure 10(b)) for uplink and downlink, respec-

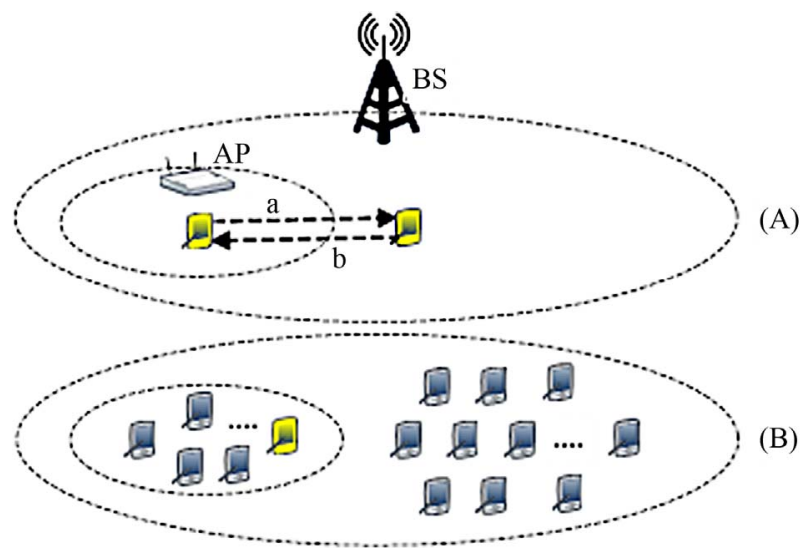

Figure 8. Simulation Scenarios for Evaluating Proposed VHM.

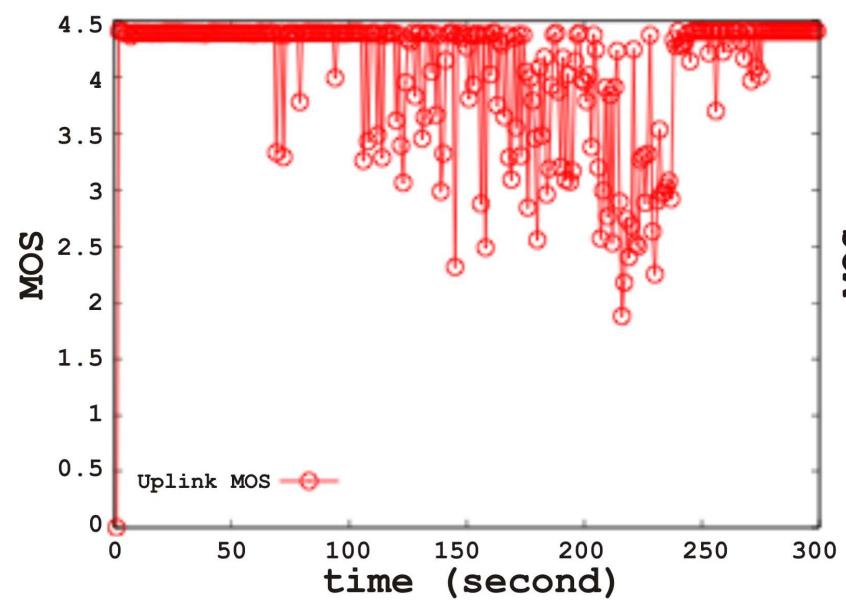

(a) Uplink MOS tively. MOS is decreased sometimes below 3.6 at simulation time of 200 seconds. This is mainly because the MN located in the edge of $802.11 \mathrm{~g}$ network at 200 seconds where link condition is degraded and also effect of fading model introduced in the simulation scenario.

Figure 11 shows the average MOS, as a function of distance between BS and AP. This figure shows that as an increase of distance between AP and BS, the average uplink and downlink MOS (when an MN moves from $802.11 \mathrm{~g}$ to $802.16 \mathrm{e}$ and vise versa) are gradually decreased. This is mainly because an overlap area between $802.11 \mathrm{~g}$ and $802.16 \mathrm{e}$ becomes less as the increase of distance between AP and BS.

Since our proposed VHM can be applied in a situation where a network cell of 802.16 overlays a network cell of $802.11 \mathrm{~g}$, lessen overlap area between two networks will affect the VoIP call quality. From Figure 11, the effecttive distance of 300 meters between AP and BS can be applied to our proposed VHM.

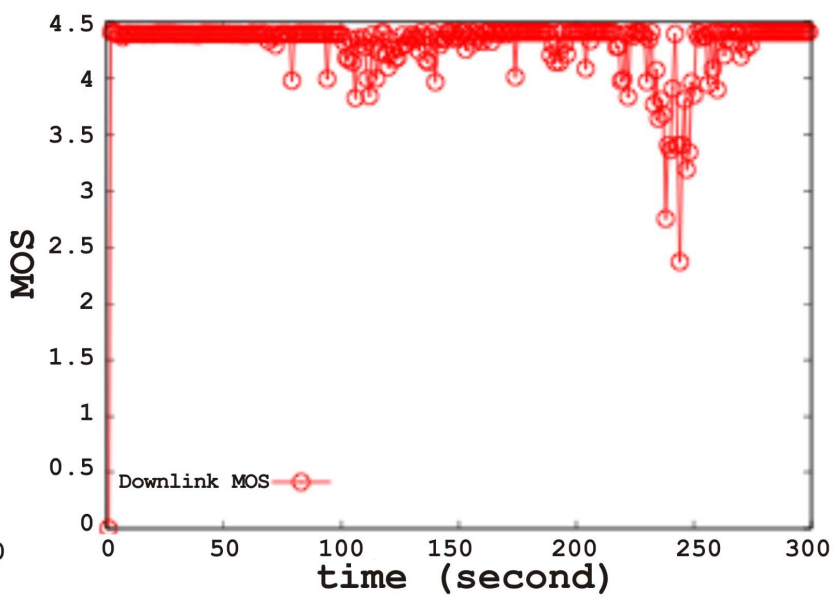

(b) Downlink MOS

Figure 9. MN moves from 802.11g to 802.16e network.

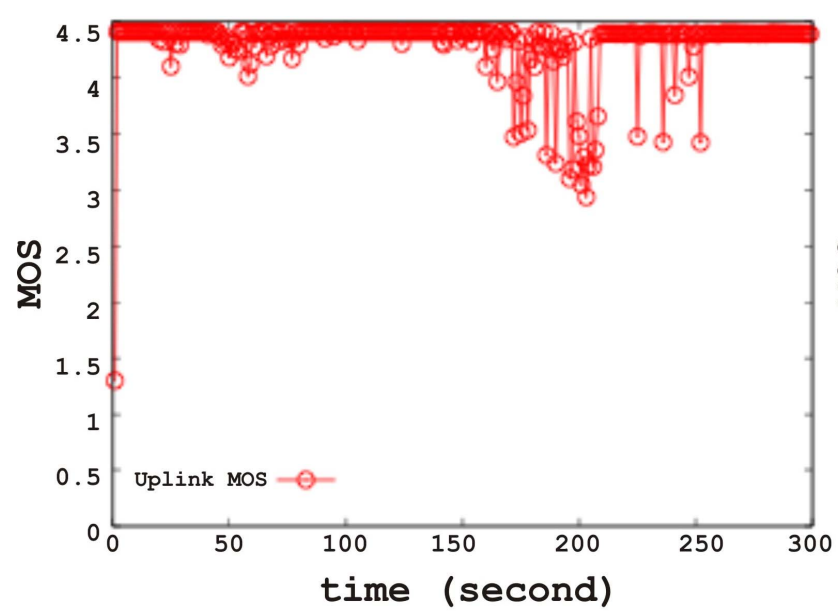

(a) Uplink MOS

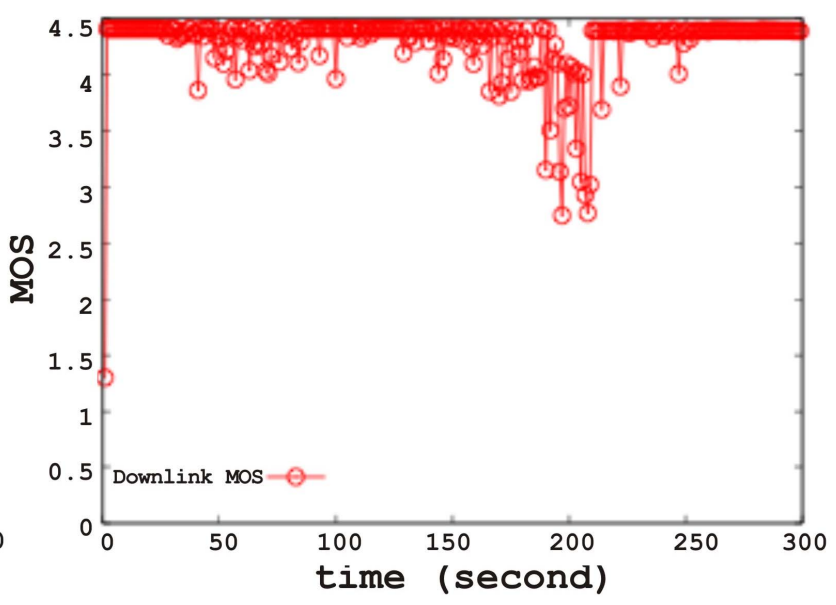

(b) Downlink MOS

Figure 10. MN moves from 802.16e to 802.11g network. 


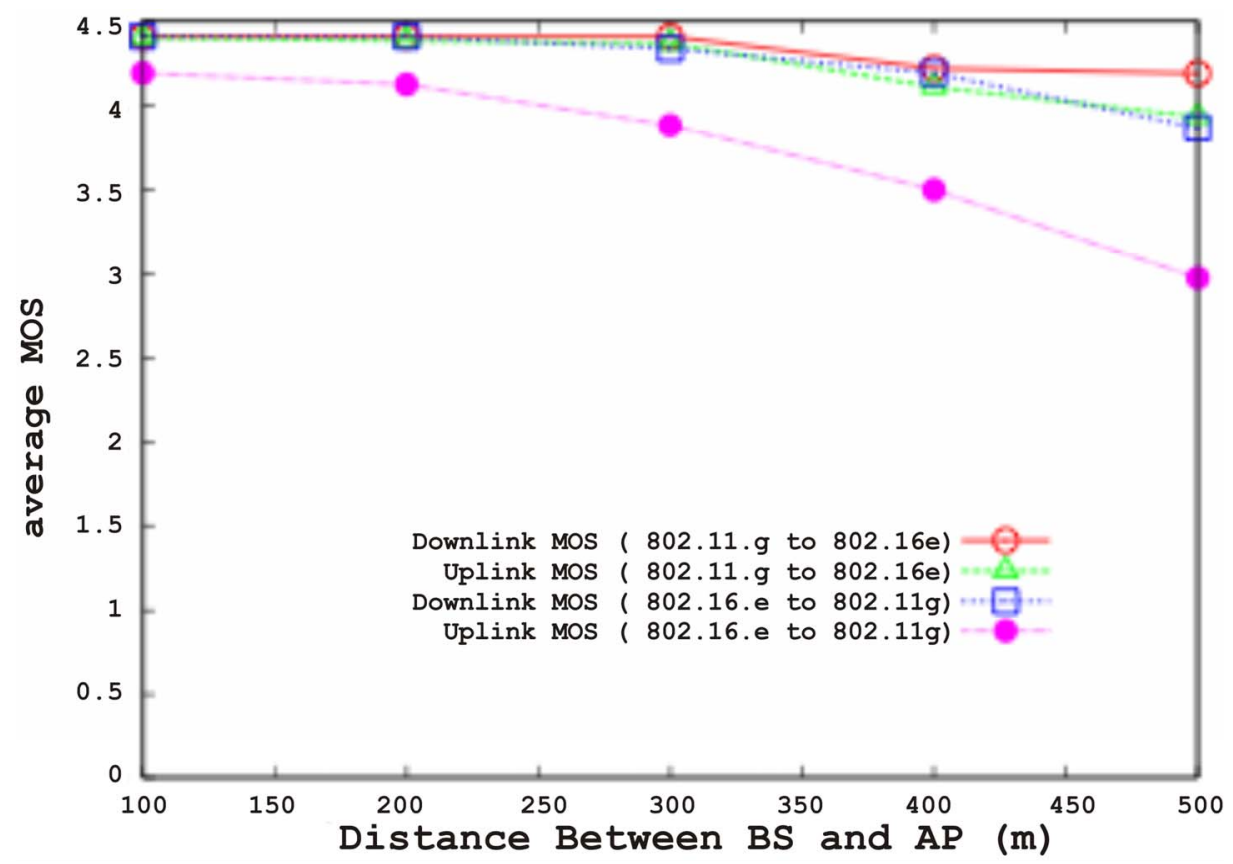

Figure 11. MOS vs. Distance between BS and AP.

\subsection{Performance Evaluation of HO Based on Congestion State HO Triggers}

This simulation experiment aims to investigate whether our proposed $\mathrm{HO}$ algorithm can initiate $\mathrm{HO}$ and decide the transmission mode according to congestion state $\mathrm{HO}$ triggers, i.e., W-RTT of $802.11 \mathrm{~g}$ IF and MN's queue length of $802.16 \mathrm{e}$ IF. First, we conducted simulation experiments to evaluate the effectiveness of our proposed VHM in a congested $802.11 \mathrm{~g}$ network. We consider the scenario depicted in Figure 8(B). At the beginning of the simulation, there are $13 \mathrm{MNs}$ randomly distributed in the vicinity of an AP (i.e., between 0 and $50 \mathrm{~m}$ ) where the access to 802.16 e networks is granted.

There is one among many MNs employing the proposed HO algorithms and it initially uses the 802.11g AP to establish a VoIP call. Every 5 seconds, a new MN establishes a VoIP connection with its $\mathrm{CN}$ via the $802.11 \mathrm{~g}$ network, so that the traffic in the $802.11 \mathrm{~g}$ network gradually increases. Simulation results show that the average uplink and downlink MOS are 4.258 and 4.249, respectively (Figures 12(a) and (b)). From these figures, the MOS is decreased sometimes below 3.6 at simulation time of 50 seconds. This is mainly because the number of $\mathrm{MN}$ reaches to maximum acceptable number of $\mathrm{MNs}$ establishing VoIP call in single AP of $802.11 \mathrm{~g}$, i.e., 10 $\mathrm{MNs}$, at the time of 50 seconds. After HO to $802.16 \mathrm{e}$, uplink MOS is decreased sometimes at initial time because uplink latency due to BW request contention slot and scheduling at BS in 802.16e. Furthermore, we also evaluate the effectiveness of our proposed VHM in a congested 802.16e network.
At the beginning of the simulation, there are $30 \mathrm{MNs}$ randomly distributed in the vicinity of $\mathrm{AP}$ and $\mathrm{BS}$. We set $30 \mathrm{MNs}$ in order to make a congested scenario in single BS (the acceptable number of MNs establishing VoIP call in the single BS of $802.16 \mathrm{e}$ is $20 \mathrm{MNs}$ as shown in Figure 4). There is one among many MNs employing the proposed VHM algorithms and it initially uses the 802.16e to establish a VoIP call. Every 3 seconds, a new MN establishes a VoIP connection with its $\mathrm{CN}$ via the $802.16 \mathrm{e}$ network, so that the traffic in the $802.16 \mathrm{e}$ network gradually increases. The simulation results show that average uplink and downlink MOS are 3.882 and 4.336, respectively (Figures 13(a) and (b)). The uplink MOS suffers degradation many times from uplink latency due to $\mathrm{BW}$ request contention and scheduling delay.

\section{Conclusions and Future Work}

In this paper, we have proposed VHM for VoIP over intermingled $802.11 \mathrm{~g}$ and $802.16 \mathrm{e}$ networks. Our proposed VHM is implemented on the transport layer of the $\mathrm{MN}$ and obtains effective $\mathrm{HO}$ triggers from the lower layer using a cross-layer approach. The VHM exploits RTS retries and W-RTT of the $802.11 \mathrm{~g}$ IF as well as CINR level and MN's queue length of the 802.16e IF as HO triggers. Our proposed method aimed to preserve VoIP quality during HO between networks with different IP subnets. Our simulation results show that our proposed VHM can preserve VoIP call quality during such HOs.

In this study, we focused on $\mathrm{HO}$ initiation and decision to switch the transmission mode, i.e., single/bi-casting 


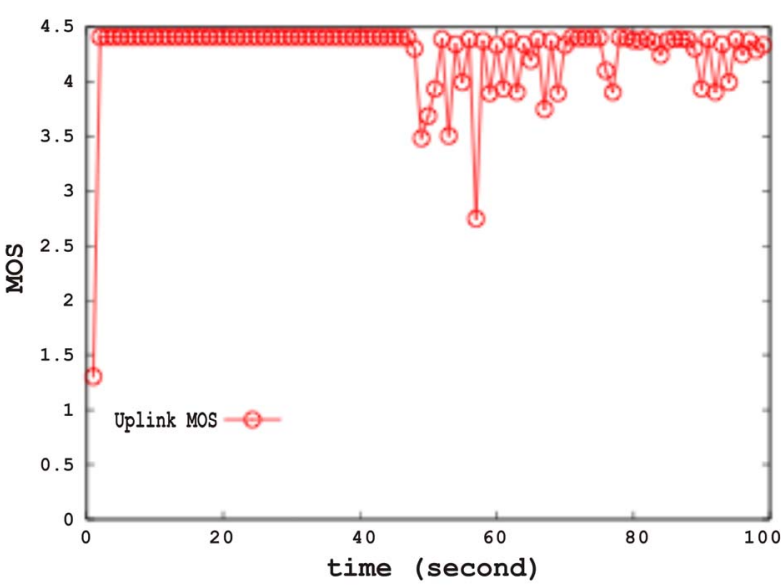

(a) Uplink MOS

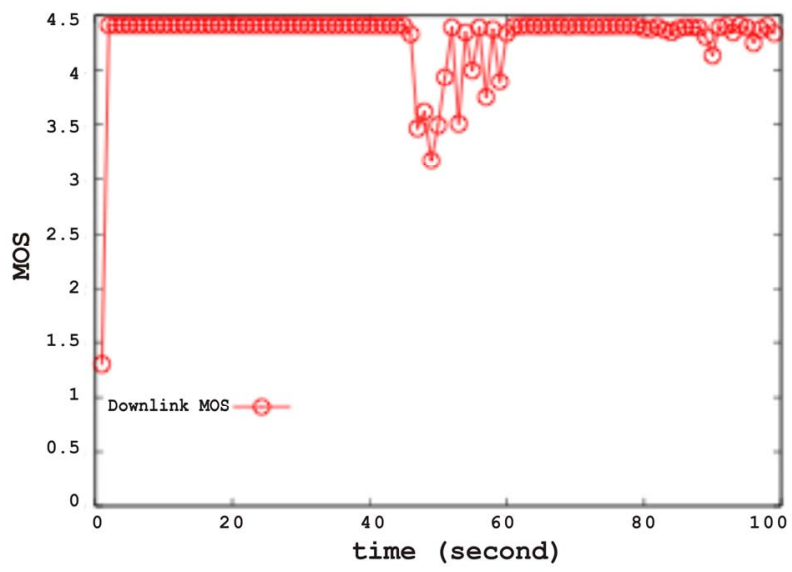

(b) Downlink MOS

Figure 12. 802.16e is congested, MN handovers to 802.11g.

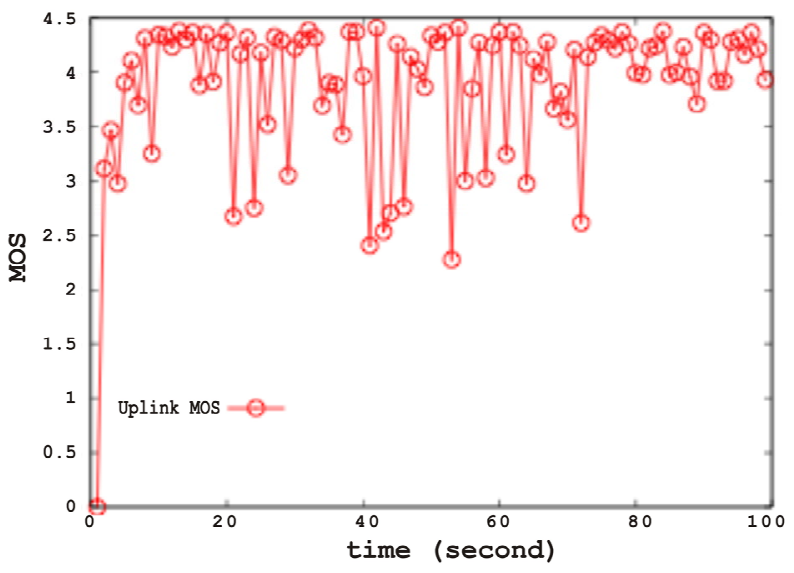

(a) Uplink MOS

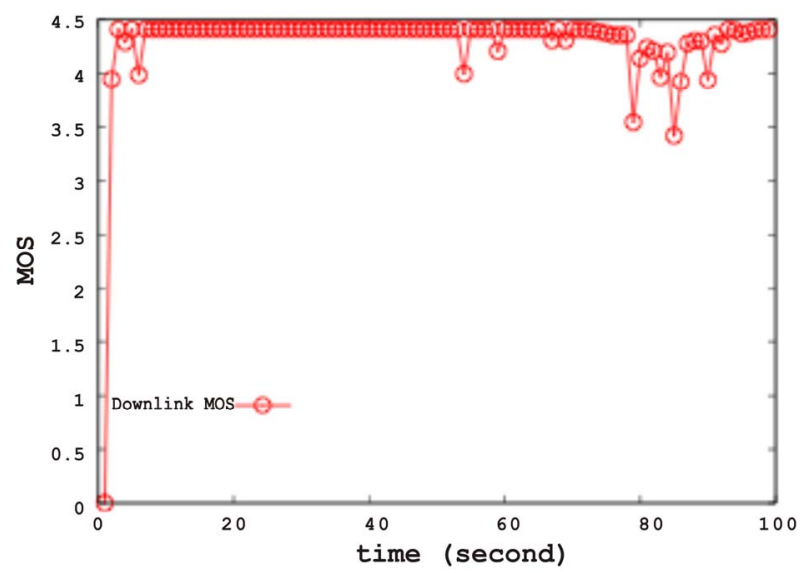

(b) Downlink MOS

Figure 13. 802.11g is congested, $\mathrm{MN}$ handovers to 802.16e.

according to $\mathrm{HO}$ triggers obtained from $802.11 \mathrm{~g}$ and $802.16 \mathrm{e}$ network. In the future, we need to extend our proposed VHM with congestion control mechanism to deal with the condition where both $802.11 \mathrm{~g}$ and $802.16 \mathrm{e}$ networks are congested.

\section{REFERENCES}

[1] A. H. Zahran and B. Liang, "Performance Evaluation Framework for Vertical Handoff Algorithms in Heteregeneous Networks," Proceedings of IEEE International Conference on Communications (ICC'05), Seoul, 16-20 May 2005, pp. 173-178.

[2] S. Mohanty and I. F. Akyilidiz, "A Cross-Layer (Layer 2 + 3) Handoff Management Protocol for Next Generation Wireless Systems," IEEE Transactions on Mobile Computing, Vol. 5, No. 10, 2006, pp. 1347-1360. doi:10.1109/TMC.2006.142

[3] H. Y. Liu, et al., "A Smart Triggering Scheme to Reduce Service Interruption during Heterogeneous Handovers," Proceedings of International Conference on Dependable Systems and Networks, Boston, 24-27 June2008.
[4] B.-J. Chang and J. F. Chen, "Cross-Layer-Based Adaptive Vertical Handoff with Predictive RSS in Heterogeneous Wireless Networks," IEEE Transaction on Vehicular Technology, Vol. 57, No. 6, 2008, pp. 3679-3692.

[5] Z. Dai, et al., "Vertical Handover Criteria and Algorithm in IEEE 802.11 and 802.16 Hybrid Networks," Proceedingds of IEEE International Conference on Communication (ICC2008), Beijing, 19-23 May 2008, pp. 24802484.

[6] A. S. Rashid, et al., "WiFi/WiMAX Heterogeneous Seamless Handover," Proceedings of 3rd International Conference on Broadband Communication, Pretoria, 23-26 November 2008, pp. 169-174.

[7] V. Gupta, et al., "IEEE 802.21 Tutorial," 2006. http://www.ieee802.org/21/Tutorials/802\%2021-IEEE-Tu torial.ppt

[8] M. Niswar, et al., "Handover Management for VoWLAN Based on Estimation of AP Queue Length and Frame Retries," IEICE Transaction on Information and System, Vol. E92-D, No. 10, 2009, pp. 1847-1856.

[9] S. Kashihara and Y. Oie, "Handover Management Based on the Number of Data Frame Retransmissions for 
VoWLAN," Elsevier Computer Communications, Vol. 30, No. 17, 2007, pp. 3257-3269.

doi:10.1016/i.comcom.2007.01.014

[10] "Scalable Network Technology." http://www.scalable- networks.com/

[11] ITU-T Recommendation G.107, "The E-Model: A Com- putational Model for Use in Transmission Planning," 2005.

[12] Cole, "Voice over IP Performance Monitoring," $A C M$ SIGCOMM Computer Communication Review, Vol. 31, No. 2, 2001, pp. 9-24. 